\title{
COVID-19 e saúde mental: a emergência do cuidado
}

\author{
COVID-19 and mental health: \\ the emergence of care
}

\author{
André FARO' ${ }^{1}$ iD 0000-0002-7348-6297 \\ Milena de Andrade BAHIANO ${ }^{1}$ (iD 0000-0003-2922-713X \\ Tatiana de Cassia NAKANO² (iD 0000-0002-5720-8940 \\ Catiele REIS ${ }^{3}$ (iD 0000-0003-1307-0393 \\ Brenda Fernanda Pereira da SILVA ${ }^{1}$ (iD) 0000-0003-1139-0342 \\ Laís Santos VITTI² iD 0000-0001-7246-7476
}

\section{Resumo}

Considerando-se a situação atual mundial, marcada por importantes crises na saúde pública e, mais recentemente, a pandemia causada pela COVID-19, o presente artigo buscou reunir informações e achados de pesquisa a respeito do impacto de tais crises na saúde mental. O texto traz conceitos relacionados à problemática do novo coronavírus e analisa consequências de medidas adotadas para lidar com situações desse tipo, tais como distanciamento social, quarentena e isolamento, ao longo de três períodos distintos: pré-crise, intracrise e pós-crise. O artigo enfoca as repercussões observadas na saúde mental da população, refletindo acerca dos desfechos favoráveis e desfavoráveis dentro do processo de crise. Por fim, são apresentadas questões relacionadas à emergência do cuidado em saúde mental, tanto aquele prestado pela Psicologia, como aquele que pode ser desenvolvido pelos demais profissionais de saúde, de modo a minimizar os impactos negativos da crise e atuar de modo preventivo.

Palavras-chave: Epidemia; Intervenção na crise; Pandemia; Surtos de doenças.

$\boldsymbol{\nabla V} \mathbf{v}$

1 Universidade Federal de Sergipe, Departamento de Psicologia, Programa de Pós-Graduação em Psicologia. Av. Marechal Rondon, s/n., Conj. Rosa Elze, 49000-000, São Cristóvão, SE, Brasil. Correspondência para/Correspondence to: A. FARO. E-mail: <andre.faro.ufs@ gmail.com>.

2 Pontifícia Universidade Católica de Campinas (PUC-Campinas), Centro de Ciências da Vida, Programa de Pós-Graduação em Psicologia. Campinas, SP, Brasil.

${ }^{3}$ Centro Universitário Ages, Curso de Psicologia, Colegiado de Psicologia. Paripiranga, BA, Brasil.

Como citar este artigo/How to cite this article

Faro, A., Bahiano, M. A., Nakano, T. C., Reis, C., Silva, B. F. P., \& Vitti, L. S. (2020). COVID-19 e saúde mental: a emergência do cuidado. Estudos de Psicologia (Campinas), 37, e200074. https://doi.org/10.1590/1982-0275202037e200074 


\begin{abstract}
Considering the current global situation, marked by important public health crises and, more recently, the COVID-19 pandemic, this article aims to gather information and research findings on the impact of such crises on mental health. The text presents the main concepts related to the new coronavirus and analyzes the consequences of the measures adopted to deal with scenarios such as the current problem, which involve social distance, quarantine, and isolation, within three distinct moments: pre-crisis, intra-crisis, and post-crisis. The paper focuses on the repercussions observed on the population's mental health and discusses favorable and unfavorable outcomes within the crisis process. To conclude, it presents questions related to the emergence of mental health care provided by Psychology and other health professionals in order to reduce negative impacts of this crisis and act in a preventive function.
\end{abstract}

Keywords: Epidemics; Crisis intervention; Pandemics; Disease outbreak.

A preocupação com a saúde mental da população se intensifica durante uma grave crise social. A pandemia da Coronavirus Disease 2019 (COVID-19) pode ser descrita como uma dessas crises, a qual tem se caracterizado como um dos maiores problemas de saúde pública internacional das últimas décadas, tendo atingido praticamente todo o planeta (World Health Organization [WHO], 2020a). Um evento como esse ocasiona perturbações psicológicas e sociais que afetam a capacidade de enfrentamento de toda a sociedade, em variados níveis de intensidade e propagação (Ministério da Saúde do Brasil, 2020a). Esforços emergenciais de diferentes áreas do conhecimento - dentre elas a Psicologia - são demandados a propor formas de lidar com o contexto que permeia a crise.

A COVID-19, nome da síndrome respiratória ocasionada pelo novo coronavírus, foi inicialmente detectada em 2019 na cidade de Wuhan, capital da província da China Central. Ela atingiu as pessoas em diferentes níveis de complexidade, sendo os casos mais graves acometidos de uma insuficiência respiratória aguda que requer cuidados hospitalares intensivos - incluindo o uso de ventilação mecânica (Centers for Disease Control and Prevention [CDC], 2020b). Até meados de abril de 2020 haviam sido contabilizados mais de dois milhões de casos notificados e quase 150 mil mortes no mundo, com os Estados Unidos (EUA) liderando a quantidade de óbitos (mais de 25 mil). A facilidade de propagação, a falta de conhecimento sobre o vírus e o aumento exponencial do número de contágios fizeram com que a Organização Mundial de Saúde (OMS) elevasse a doença ao status de pandemia em março de 2020 (WHO, 2020a).

A China alcançou o status de único país a começar a viver os efeitos posteriores da crise em abril de 2020, passando a atuar no auxílio às nações que ainda estavam em estágios iniciais da pandemia, a exemplo da Itália e Estados Unidos. O Brasil, na terceira semana de abril de 2020, havia ultrapassado trinta mil casos confirmados, com mais de 1.500 mortes e taxa de mortalidade em torno de 5,5\%. O país vivia o período inicial da crise, com elevado grau de subnotificação - principalmente pela baixa quantidade de testes realizados na população até então - e ainda buscava reestruturar seu sistema de saúde para comportar o maior número de doentes possível (Ministério da Saúde, 2020a). Ressalta-se que nem mesmo a China descarta a possibilidade de uma segunda onda de infecções, talvez mais abrangente que a primeira, seja devido ao relaxamento com os cuidados relacionados ao isolamento, seja pela possibilidade de recontágio, situação ainda em estudo (Bikbov \& Bikbov, 2020).

A taxa de mortalidade da COVID-19 tem se mostrado superior à das gripes periódicas, a exemplo do grupo Influenza (L. Li et al., 2020). A falta de imunização frente a esse vírus e, principalmente, sua elevada facilidade de propagação têm caracterizado a emergência do problema de saúde pública internacional (Sohrabi et al., 2020). Já é uma marca da pandemia do novo coronavírus que fração significativa das pessoas com sintomas da COVID-19 tende a demandar atendimento quase que simultaneamente, de modo a 
superlotar os sistemas de saúde. Isso tem ocorrido em virtude do medo disseminado a respeito da doença e/ ou da severidade de alguns sintomas - febre e falta de ar, principalmente -, o que provoca dificuldades, em cadeia, nos sistemas de saúde de todo o mundo (Brooks et al., 2020). Essa demanda súbita por assistência imediata sobrecarrega todos os níveis de atenção, especialmente o terciário (hospitalar, de medicina intensiva), desencadeando crises na saúde pública tanto de países em desenvolvimento quanto em países ricos, quase que ao mesmo tempo e em todos os continentes, situação sem precedentes ao longo das últimas décadas (Mahase, 2020; Peeri et al., 2020; WHO, 2020a).

O combate a situações como esta já vinha sendo motivo de debate na OMS, que, em 2007, publicou um documento ressaltando a necessidade de haver planejamento prévio por parte dos países, para o enfrentamento de crises e desastres inesperados (WHO, 2007). Cabe ainda dizer que outros estudos sobre crises em saúde pública também haviam ressaltado que os cuidados em saúde mental deveriam ser tão primordiais quanto os cuidados primários de saúde (Douglas, Douglas, \& Douglas, 2009; Park \& Park, 2020; Stevenson et al., 2009).

Além do medo de contrair a doença, a COVID-19 tem provocado sensação de insegurança em todos aspectos da vida, da perspectiva coletiva à individual, do funcionamento diário da sociedade às modificações nas relações interpessoais (Lima et al., 2020; Ozili \& Arun, 2020). Quanto à saúde mental, é importante dizer que as sequelas de uma pandemia são maiores do que o número de mortes. Os sistemas de saúde dos países entram em colapso, os profissionais de saúde ficam exaustos com as longas horas de trabalho e, além disso, o método de controle mais efetivo da doença, que é o distanciamento social, impacta consideravelmente a saúde mental da população (Brooks et al., 2020).

Desde as experiências advindas dos surtos da Severe Acute Respiratory Syndrome (SARS, Síndrome Respiratória Aguda Grave), em 2002, e do Ebola, entre 2013 e 2016 (Brooks et al., 2020), da pandemia da H1N1 em 2009 (Bajardi et al., 2011) e, atualmente, frente à COVID-19, percebe-se a necessidade de investigar e agir em questões relativas à saúde mental ao longo da crise, uma vez que a capacidade de alcançar um estado de ajustamento psicológico saudável é desafiada, dia após dia, por inúmeras adversidades não usuais nesse período (Brooks et al., 2020; Ho, Chee, \& Ho, 2020; Lima et al., 2020; Ozili \& Arun, 2020). Isso reitera a constatação de que, durante uma pandemia, é provável que seja vivenciada uma carga elevada de experiências e emoções negativas, suscitando a necessidade de cuidados psicológicos constantes desde o período inicial do problema (Ho et al., 2020; W. Li et al., 2020). Recentemente, diferentes organizações internacionais se manifestaram quanto à premência dos cuidados em saúde mental na pandemia da COVID-19 (WHO, 2020a; European Centre for Disease Prevention and Control [ECDC], 2020), cabendo ressaltar o Ministério de Saúde brasileiro, que também enfatizou a relevância dessa questão no país (Ministério da Saúde, 2020a). Portanto, fica patente a importância de profissionais da Psicologia estarem capacitados a auxiliar no desenvolvimento de modos mais saudáveis de lidar com a atual crise em saúde, colaborando em seus diferentes níveis de atuação, gestão política, avaliação epidemiológica e cuidados primários (C. Wang et al., 2020).

Para contribuir com esse desafio, este trabalho reuniu, de modo breve, informações e achados de pesquisa a respeito da saúde mental frente a crises em saúde pública, como surtos e pandemias, trazendo para o centro da análise a COVID-19. Pretende-se, ainda, apresentar uma noção de emergência do cuidado em saúde mental, tanto aquele prestado pela Psicologia, como aquele que pode ser desenvolvido pelos demais profissionais de saúde. Para tanto, o texto a seguir está subdividido em quatro partes: (a) Distanciamento social, quarentena e isolamento: conceitos e recomendações; (b) A noção de crise no contexto de pandemia e repercussões em saúde mental; e (c) A emergência do cuidado na crise da COVID-19. Nas considerações finais (d) são analisados os impactos gerais da pandemia na saúde mental e como isso pode repercutir em um cenário futuro. 


\section{Distanciamento social, quarentena e isolamento: conceitos e recomendações}

No âmbito da prevenção em saúde, algumas medidas são tomadas para proteger a comunidade da exposição ao risco de doenças contagiosas. O distanciamento social ou distanciamento físico implica a manutenção de uma distância espacial - cerca de dois metros - entre o indivíduo e outras pessoas, quando fora de casa (CDC, 2020a; ECDC, 2020). Isso resulta, por exemplo, na recomendação de não se reunir em grupos e evitar lugares cheios e aglomerações. Essa é uma medida que vem sendo executada pelos países para evitar o contágio.

Outras medidas utilizadas são a quarentena e o isolamento. Esses dois termos, que muitas vezes são usados como sinônimos, possuem significados e objetivos distintos (Brooks et al., 2020). A quarentena busca separar e restringir a circulação de pessoas que foram expostas a uma doença contagiosa, visando a observar se estas ficarão doentes. Já o isolamento diz respeito à separação de pessoas doentes, infectadas por alguma doença transmissível, como a COVID-19, dos não doentes (CDC, 2020a). No Brasil, a Portaria $\mathrm{n}^{\circ}$ 454 (Ministério da Saúde, 2020c) declarou estado de transmissão comunitária do novo coronavírus em 20 de março de 2020, o que fez entrar em vigor a Lei da Quarentena, Lei n 13.979 (Presidência da República, 2020), com o objetivo de evitar a contaminação e propagação da COVID-19.

A quarentena é fundamental para atenuar o contágio. O pressuposto de manter as pessoas sem contato com outras pessoas busca diminuir a probabilidade de contaminação e, consequentemente, a procura por serviços de saúde e o número de óbitos. Trata-se de uma medida usada há muitos anos para evitar a disseminação de doenças contagiosas (Brooks et al., 2020). Apesar dos benefícios que traz, em função da contenção da doença, a quarentena implica, muitas vezes, a vivência de situações desagradáveis que podem ocasionar impactos na saúde mental dos envolvidos. Alguns estressores na quarentena são: necessidade de afastamento de amigos e familiares, incerteza quanto ao tempo de distanciamento (Brooks et al., 2020), tédio (Barari et al., 2020), medo (Lima et al., 2020) e outros. Em estudos realizados em situações pandêmicas, como é o caso da COVID-19 e da SARS, constatou-se que alguns transtornos mentais comuns podem ser desencadeados pela quarentena, a exemplo dos transtornos de ansiedade (Barari et al., 2020; Lima et al., 2020) e depressão (Pancani, Marinucci, Aureli, \& Riva, 2020) e indícios de aumento do comportamento suicida (Barbisch, Koenig, \& Shih, 2015).

Frente às repercussões psicológicas que o distanciamento social pode promover, algumas medidas podem ser tomadas para que ele se torne o menos danoso possível. O tempo mínimo indicado para a quarentena tem sido de duas semanas, que é o período de incubação do vírus da COVID-19. Ainda que esse período seja estendido, é importante que ele dure, dentro do necessário, o mínimo possível para ser menos nocivo à saúde mental (Brooks et al., 2020). Outra estratégia para que a quarentena seja menos prejudicial é a informação. Deve-se comunicar às pessoas o que está acontecendo e os motivos, explicando por quanto tempo isso pode durar, mantendo-as informadas sobre a importância de ficar em casa (Pancani et al., 2020). Por fim, a manutenção das redes de apoio social durante a quarentena também é essencial para a saúde mental, já que a ruptura das conexões sociais e físicas é um importante facilitador de impactos psicológicos negativos (CDC, 2020a; ECDC, 2020).

Em surtos e pandemias ocorridos anteriormente, muitas cidades foram mantidas em quarentena. É o caso de áreas da China e Canadá durante a SARS em 2003, bem como de países da África Ocidental com o Ebola, em 2014 (Brooks et al., 2020). Na pandemia da COVID-19, a maioria dos países afetados tem adotado o regime de quarentena para conter o contágio. Neste trabalho serão destacadas as ações tomadas pela China, Itália, Espanha, Estados Unidos e Brasil em função dos cenários vividos por esses países frente à pandemia, no começo de abril de 2020. 
A China foi o primeiro país a exibir casos do novo coronavírus, em dezembro de 2019. Em meados de abril de 2020 já havia passado pelo período agudo da pandemia, mas a iminência de uma segunda onda do coronavírus, que evidenciava a possibilidade de retorno da transmissão comunitária, trouxe novamente à pauta a alternativa de decretação de um novo regime de quarentena (Bikbov \& Bikbov, 2020). Embora já tivesse ocorrido o pico de maior disseminação do vírus e o país estivesse experimentando as diversas repercussões da primeira onda da COVID-19, o governo chinês manteve medidas de prevenção e controle. Foram continuadas as restrições relacionadas à importação, exportação de produtos, mobilidade dentro do país e entrada de estrangeiros, sendo também desenvolvidas ações para a melhoria da assistência e cuidados em saúde mental (National Health Commission of China [NHC], 2020b; WHO, 2020b).

Os casos na Itália e na Espanha tiveram início no fim de janeiro de 2020, de modo que ambos os países experimentaram o pico da pandemia em período similar, no início de abril de 2020. A Itália teve um elevado número de mortes, com mais de vinte mil óbitos até o final da primeira quinzena de abril (Coronavirus Resource Center, 2020). O país determinou, em 25 de março de 2020, medidas como quarentena obrigatória e aplicou multas para quem a desobedecesse. Aglomerações foram proibidas e eventos públicos cancelados (Ministero della Salute, 2020). A alta taxa de mortalidade do país pareceu associar-se ao fato de que quase um quarto de sua população possuía 65 anos ou mais, especialmente na região da Lombardia, que concentrou cerca de metade das mortes (Onder, Rezza, \& Brusaferro, 2020).

Na Espanha, com o objetivo de conter o aumento de casos da COVID-19, o governo decretou estado de alerta em 14 de março de 2020. O Decreto 463/2020 propôs-se a proteger a saúde e a segurança dos cidadãos, conter a progressão da doença e fortalecer o sistema público de saúde. Algumas medidas estabelecidas foram: limitação da circulação de pessoas, sendo possível sair apenas para atividades essenciais; suspensão de atividades educativas presenciais e outras (Agencia Estatal Boletín Oficial del Estado [BOE], 2020a). O decreto anterior foi estendido, seguindo em vigor até o final de primeira quinzena de abril de 2020 através do Decreto 487/2020 (BOE, 2020b). O país teve mais de 180 mil casos e mais de 18 mil óbitos até então (Coronavirus Resource Center, 2020). Salienta-se que a adoção tardia de medidas mais rígidas para contenção do contágio foi um dos motivos pelos quais a situação na Espanha quanto à COVID-19 foi avaliada como grave.

Apesar de, no final de março de 2020, ter se iniciado, nos EUA, a propagação do novo coronavírus, o contágio e acometimento pela doença avançou bastante num período curto: quase 600 mil casos e 25 mil mortes até o final da primeira quinzena de abril de 2020 (Coronavirus Resource Center, 2020), especialmente na cidade de Nova lorque. Recomendações foram feitas para que as pessoas seguissem as orientações das autoridades, permanecessem em casa, evitassem aglomerações e respeitassem o distanciamento social e a quarentena, na tentativa de conter a transmissão descontrolada da doença (CDC, 2020a). Assim como ocorreu na Espanha, a demora para a adoção de medidas de contenção do vírus parece ter agravado a situação do país, o que fez com que, na segunda semana de abril, os EUA assumissem o maior número absoluto de casos confirmados e óbitos no mundo.

O Brasil, na primeira semana de abril de 2020, encontrava-se em Emergência de Saúde Pública de Importância Nacional. O estado de transmissão comunitária foi declarado em 20 de março de 2020 pela Portaria n 454 (Ministério da Saúde, 2020c). Para conter a transmissibilidade da COVID-19, adotou-se o isolamento domiciliar daqueles com sintomas respiratórios e dos residentes no mesmo endereço, ainda que assintomáticos, pelo período de catorze dias.

Em rastreamento realizado em 12 de abril de 2020, aproximadamente 46,2\% da população brasileira havia aderido à quarentena, tendo sido atingido o pico de adesão em 22 de março, com 69,3\% (recomendável: a partir de 70,0\%) (Inloco, 2020). Após esse período, observou-se um declínio da adesão nas semanas seguintes, especificamente no começo de abril, o que demonstrou que parte da população deixou de atender 
integralmente às recomendações de quarentena. Esse fenômeno parece ter sido reforçado pela propagação de informações ambíguas ou falsas em relação à COVID-19, o que ocorreu, inclusive, por parte de agentes governamentais. Frente a isso, o Ministério da Saúde brasileiro reiterou as recomendações de distanciamento e reclusão ao longo do mês de abril e para os dois meses seguintes, buscando convergência com as orientações da OMS e outros órgãos internacionais (CDC, 2020a; ECDC, 2020).

\section{A noção de crise no contexto de pandemia e repercussões em saúde mental}

Entender como se apresenta uma crise em termos de estágios de evolução do problema de saúde pública é importante para preparar profissionais de saúde e a população em geral. Isso se dá pois é necessário implementar estratégias de controle e alertar a população sobre riscos imediatos e continuados, visto que a adesão a medidas preventivas vai depender de como as pessoas percebem essa ameaça (WHO, 2020b). É recomendado, portanto, reduzir a ambiguidade das informações, especialmente as que podem gerar sintomas relacionados à ansiedade e estresse (Brooks et al., 2020). Logo, avaliar uma crise como momentos encadeados e progressivos pode colaborar para o entendimento de especificidades de fatores estressores relativos à situação-problema e o cuidado que deve ser prestado.

Nesta seção utiliza-se uma noção de crise que pode ser dividida, didaticamente, em três momentos: pré-crise, intracrise e pós-crise. Para cada um deles é possível estimar as repercussões mais observadas na saúde mental, as quais seriam produto de movimentos de exposição e proteção dos indivíduos ao longo do período de emergência em saúde pública. A seguir são apresentados conceitos para cada momento da crise, aspectos favoráveis e desfavoráveis ao enfrentamento coletivo e/ou individual de cada um deles, bem como desfechos em saúde mental que tendem a ocorrer em contextos dessa natureza.

\section{Pré-crise}

A primeira fase é o momento no qual são passadas, para a população em geral, as principais informações quanto à existência do problema de saúde pública e às formas de contágio, transmissão ou desenvolvimento da doença e seus sintomas, além das consequências relacionadas ao fator de adoecimento. No caso da COVID-19, o primeiro estágio se associa às informações sobre seu impacto inicial, sendo feitas recomendações de distanciamento social. Além disso, difundiram-se informações sobre a mortalidade mais alta em idosos, apesar da baixa letalidade geral da doença, as características em parte similares a gripes sazonais e a transmissão por contato direto ou mediado com indivíduos doentes (WHO, 2020a).

Na fase pré-crise, o planejamento é essencial. Esse momento deve envolver a avaliação de riscos, a qual deve ser atualizada regularmente, baseando-se na consulta a orientações técnicas fornecidas pela OMS sobre a COVID-19. Outra ação recomendada envolve a organização dos recursos financeiros e insumos, bem como a implantação de medidas focadas no controle da transmissão do vírus, como o distanciamento social (WHO, 2020a). Durante uma pandemia, é importante aumentar a conscientização da população-alvo sobre a patologia, de modo a diminuir a ansiedade social e estimular mudanças comportamentais que poderão ajudar no controle da doença (WHO, 2020b). Logo, a comunicação é útil na gestão de uma emergência sanitária que gera preocupação, ansiedade e pânico na sociedade (WHO, 2020b).

Na COVID-19, otimismo irrealista e emoções negativas podem ser desencadeadas, como consequência da influência midiática em torno da pandemia (Brooks et al., 2020). Tanto o otimismo irrealista - que seria a crença de que tudo dará certo, independentemente das ações dos atores envolvidos - quanto as emoções negativas - a exemplo da tristeza, angústia e medo - podem acentuar previsões distorcidas sobre a saúde. Nesses casos, tende-se a observar a percepção de menor risco de contaminação, com a adoção de comportamentos 
indesejáveis, como a quebra do distanciamento social - ou mesmo estimular as preocupações exageradas com a ameaça percebida, como o acúmulo de materiais de higiene e equipamentos de proteção, causando escassez para os profissionais da saúde (Raude et al., 2020; van Bavel et al., 2020).

Quanto aos desfechos em saúde mental, durante a pré-crise da COVID-19 na Itália, a duração da quarentena e/ou distanciamento social assim como a inadequação do espaço utilizado aumentaram a ocorrência de sintomas depressivos (Pancani et al., 2020). O estresse agudo é outro fator presente nessa fase. Em pesquisa com pessoas de mais de quarenta países, como Espanha, Colômbia e Filipinas, observou-se que 'maior preocupação em relação ao contágio', 'gênero feminino' e 'faixas etárias mais jovens' se mostraram preditores significativos para maior nível de estresse (Limcaoco, Mateos, Fernandez, \& Roncero, 2020). Sintomas depressivos e aumento de comportamentos relacionados à dependência de substâncias, como o tabagismo, também ocorreram a longo prazo, conforme apontado em estudo com profissionais da saúde de Taiwan que cuidaram de pacientes com suspeita de SARS (Lung, Lu, Chang, \& Shu, 2020).

\section{Intracrise}

O período intracrise, ou fase aguda, é o momento no qual o problema de saúde se instala, com a constatação da gravidade e vulnerabilidade ao adoecimento, e o reconhecimento do risco eventual de contágio. Na crise desencadeada pela COVID-19, especificamente no intracrise, já se pode visualizar a alta quantidade e a simultaneidade dos casos suspeitos e confirmados, situação que tende a sobrecarregar o sistema de saúde, levando ao colapso da assistência e agravando o cenário da pandemia (Weissman et al., 2020). Em situações de aceleração descontrolada do contágio, as internações e os óbitos pela COVID-19 tendem a ser frequentes, com capacidade cumulativa elevada e em prazo bastante curto, desde o início de apresentação dos sintomas ou agravamento do quadro. Esse fenômeno provoca intensas repercussões no funcionamento social e tem potencial de impactar severamente a saúde mental da sociedade, a exemplo do colapso dos sistemas de saúde e funerário que está acontecendo em abril de 2020 no Equador (Coronavirus Resource Center, 2020). Some-se a esse quadro o fato de que, devido ao alto potencial de contágio, familiares em todo o mundo não estão podendo velar nem enterrar seus parentes acometidos pelo vírus. Tal situação tem gerado uma série de questões emocionais, além daquelas que comumente já acompanham a perda.

$\mathrm{Na}$ China, no período intracrise, houve uma intensa proposição de estratégias voltadas para a redução do número de infectados e de mortes. Especialmente na cidade de Wuhan e áreas próximas, foram adotadas diferentes medidas, como o tratamento ativo dos doentes, o isolamento e observação médica das pessoas próximas aos infectados e a construção de novos hospitais, dentre outras (WHO, 2020b). Na Itália foram implementadas medidas extraordinárias para tentar controlar a transmissão do vírus, a exemplo do distanciamento social em caráter emergencial, especialmente na região da Lombardia (Remuzzi \& Remuzzi, 2020). No final de março e início de abril de 2020, os hospitais italianos de algumas localidades não mais dispunham de recursos ou insumos suficientes para atender à demanda de enfermos (leitos em Unidades de Terapia Intensiva, aparelhos de ventilação artificial e/ou equipamentos de proteção individual) nem possuíam equipes de profissionais de saúde em número adequado. Isso levou à desassistência de casos severos, caracterizando o colapso do sistema de saúde em algumas regiões. Somou-se a esse quadro o fato de que, dentre os trabalhadores da área de saúde, muitos estavam trabalhando em mais de um turno, haviam sido infectados e, consequentemente, afastados (Remuzzi \& Remuzzi, 2020).

Ainda que a maior parte dos países tenha adotado como principal estratégia o lema de "salvar vidas", o desconhecimento de aspectos relacionados ao intracrise da COVID-19 (tratamento complexo de casos graves e escassez mundial de insumos de proteção) tem gerado impacto negativo na saúde, tanto física quanto mental, de todos os envolvidos com a assistência à saúde (Horton, 2020). Pesquisas voltadas à investigação 
dos efeitos do novo coronavírus sobre a saúde mental também têm indicado o aumento de quadros como depressão, ansiedade, estresse, transtorno do pânico, insônia, medo e raiva em diferentes países (Duan \& Zhu, 2020; G. Wang, Zhang, Zhao, Zhang, \& Jiang, 2020; Yang et al., 2020).

Dentre os pacientes confirmados ou com suspeita da COVID-19, são comuns relatos de tédio, solidão e raiva, juntamente com seus familiares próximos, os quais também têm sido foco de atenção, dado o fato de que alguns têm apresentado sintomas relacionados ao estresse pós-traumático (Xiang et al., 2020). Com esses grupos, sintomas somáticos, insônia, ansiedade, raiva, ruminação, diminuição da concentração, mau humor e perda de energia devem receber atenção especial nos cuidados de saúde mental (Park \& Park, 2020). Soma-se a isso o aguçamento das preocupações consigo e com os outros durante a epidemia, o que passa a ser uma rotina cada vez mais exigente durante o intracrise. Isso tende a elevar carga emocional, física e de papéis sociais, facilitando o desencadeamento, agravamento ou recidiva de transtornos mentais ou doenças físicas (Brooks et al., 2020).

Neste momento da crise percebe-se que as pandemias não são apenas um fenômeno biológico, pois afetam indivíduos e a sociedade em vários níveis, causando diversas perturbações. Por isso, durante o período intracrise ou mesmo desde meados do pré-crise, em diversos países, instituições médicas e universidades abriram plataformas online para fornecer aconselhamento psicológico para pacientes, familiares que estão em quarentena e outras pessoas afetadas pela pandemia, visando minimizar o pânico e separação da família (Duan \& Zhu, 2020). Movimento similar foi adotado no Brasil pelo Conselho Federal de Psicologia do Paraná, por meio de uma Nota Técnica (CRP-PR n. 001/2020), orientando psicólogos sobre o atendimento dentro das políticas públicas de atenção à saúde e em instituições privadas (Conselho Regional do Paraná, 2020).

\section{Pós-crise}

O terceiro momento da crise pode ser compreendido como uma fase de reconstrução social. Após o declínio do número de novos casos e a diminuição da transmissão comunitária, as medidas de distanciamento social são reduzidas e o surto de contaminação tende a estar sob controle, ainda que não seja necessariamente inexistente. As pessoas começam a retomar as atividades habituais, há o retorno gradual do funcionamento das instituições e comércio, além de um menor nível de exigência de proteção contra o contágio. Na pandemia da COVID-19, até o dia 15 de abril de 2020, apenas a China havia iniciado essa fase, conforme os casos de transmissão comunitária, internações e mortes começaram a se reduzir.

Apesar da progressiva retomada da rotina diária em curto prazo, uma série de consequências da pandemia demanda prazos médio e longo para serem revertidas. Em pesquisa realizada na crise da COVID-19, verificou-se que, dentre 1.210 participantes, 53,0\% apresentaram sequelas psicológicas moderadas ou severas, incluindo sintomas depressivos (16,5\%), ansiedade (28,8\%) e estresse de moderado a grave $(8,1 \%)$ (C. Wang et al., 2020). Os maiores impactos foram verificados no sexo feminino, estudantes e pessoas com algum sintoma relacionado à COVID-19, bem como naqueles que julgavam sua saúde como ruim. Outro estudo no pós-crise, realizado com cerca de 52 mil chineses, detectou que mulheres, pessoas com mais de sessenta anos, com maior nível educacional e migrantes foram mais vulneráveis ao estresse, ansiedade, depressão, fobias específicas, evitação, comportamento compulsivo, sintomas físicos e prejuízos no funcionamento social (Qiu et al., 2020).

Outra consequência observada no pós-crise foi (ou mesmo "está sendo") a discriminação e isolamento vivenciados por estudantes chineses, devido ao fato de serem considerados portadores em potencial do novo coronavírus. Dentre os participantes, foram encontrados indícios de maior vulnerabilidade ao estresse, ansiedade e medo persistentes (Zhai \& Du, 2020). Atrelado a esse fenômeno, há preocupação quanto à estigmatização das pessoas que estão sendo tratadas ou mesmo foram curadas pela COVID-19 (ECDC, 2020), 
devendo-se evitar termos como "vítima da COVID-19", "COVID positivo", "contaminado pela COVID" ou "caso de COVID-19", pois denotam coisificação e agregam maior sofrimento. É preferível o uso de expressões como "pessoa que foi diagnosticada com a COVID-19", "pessoa que está com a COVID-19" ou expressões similares que não privilegiem a doença em detrimento do indivíduo. Caso a discriminação se consolide como resposta social, o receio pelo duplo dano relativo à COVID-19 (adoecimento e estigmatização) pode atrasar a busca pelo diagnóstico e cuidado apropriado, de modo a produzir uma ameaça adicional ao controle efetivo da doença na sociedade.

\section{A emergência do cuidado na crise da COVID-19}

Usualmente, boa parte dos esforços das autoridades de saúde pública e dos veículos de comunicação durante as epidemias tem envolvido a compreensão dos efeitos físicos e biológicos da doença, revelando pouca, ou quase nenhuma, atenção às questões da saúde mental (Ho et al., 2020). Assim como trabalhado ao longo deste texto, não se pode minimizar as repercussões psicológicas que o cenário geral da pandemia causa sobre indivíduos em particular, grupos com características de vulnerabilidade específicas e a sociedade como um todo, visto que o impacto na saúde mental, muitas vezes, se torna um fator notavelmente limitante para que o próprio país supere uma crise como a da COVID-19 (Cullen, Gulati, \& Kelly, 2020; Ho et al., 2020; WHO, 2020a). Além das múltiplas implicações que envolvem o processo de enfrentamento e contenção de um surto pandêmico, é importante garantir à população uma assistência apropriada em saúde mental, englobando ações voltadas à minoração do sofrimento mental ao longo da crise (Cullen et al., 2020; Duan \& Zhu, 2020).

Com o objetivo de reduzir os danos psicológicos causados pela epidemia e promover estabilidade social, a China, por exemplo, publicou uma diretriz que instituiu níveis de atenção psicológica para o enfrentamento da COVID-19. As recomendações foram agrupadas em quatro níveis de populações-alvo (NHC, 2020a). O nível 1 inclui pacientes hospitalizados com infecção confirmada ou condição física grave para a COVID-19, profissionais de saúde de primeira linha e equipe administrativa. O nível 2 se refere às pessoas em isolamento por terem alguma proximidade com indivíduos confirmados para a doença, além daqueles em quarentena por terem tido contato com pessoas suspeitas de infecção. Já na população de nível 3 estão os indivíduos que tiveram contato próximo com os níveis 1 ou 2, ou seja, familiares, colegas, amigos e equipes de resgate. Por fim, o nível 4 é composto pela população em geral, que não está nem na linha de frente e nem em medidas de isolamento ou quarentena; ou seja, são aquelas para as quais se recomenda o distanciamento social (NHC, 2020a). A intervenção proposta se direciona prioritariamente à população de primeiro nível, mas com foco gradual de expansão do cuidado psicológico para os outros níveis, alcançando-se, por fim, a população em geral.

Quanto aos cuidados emergenciais de atenção psicológica que foram propostos pela diretriz, o nível 1 tem prioridade porque nele se encontram, especialmente, as pessoas mais vulneráveis ao risco de adoecimento físico e mental. Além do tratamento medicamentoso, os cuidados recomendados a esse grupo se focam no apoio psicológico do paciente, com avaliação oportuna para condutas autolesivas e risco de suicídio. Reforço das informações inerentes à importância do isolamento e encorajamento da confiança dos pacientes para a recuperação também são importantes nessa fase. Aos profissionais da linha de frente e equipe administrativa sugere-se, antes de se iniciarem os trabalhos, treinamentos e entrevistas preventivas focadas na gestão do estresse, regulação das emoções e encorajamento para a busca de ajuda psicológica diante da dificuldade de lidar com as emoções e a adversidade da situação (NHC, 2020a).

O cuidado do nível 2 volta-se às pessoas que se encontram em isolamento ou quarentena. A intervenção realizada pelos profissionais da saúde mental nesse público deve ser realizada precocemente, visando a reduzir 
a angústia, tensão e estresse relativos ao real ou potencial adoecimento, buscando também estimular a adesão às medidas de proteção necessárias para si e contra a propagação do vírus. No nível 3, constituído por pessoas que tiveram contato com indivíduos dos níveis anteriores, enfatiza-se a observância de comportamentos de inquietação, ansiedade durante a espera e dificuldades para manter as regras de distanciamento social. É incentivada a utilização de serviços de atendimento online para a redução de sintomas psicológicos. No nível 4, voltado ao público em geral, a intervenção é focada na divulgação de informações baseadas em evidências; esclarecimentos sobre atendimentos em saúde mental disponibilizados pelas redes de atenção básica, psicológica e psicossocial; orientações sobre a forma de se adaptar às mudanças impostas pela crise pandêmica; e não discriminação dos pacientes diagnosticados ou suspeitos da COVID-19. Também é reforçado que métodos de enfrentamento não saudáveis sejam evitados, a exemplo do consumo de álcool, tabaco e outras drogas (NHC, 2020a).

Independentemente da fase da crise em que se encontre uma localidade, espera-se que tenda a subir a demanda por cuidados em saúde mental dos pacientes diagnosticados com a COVID-19, de seus familiares, de grupos com vulnerabilidades específicas (por exemplo, idosos e pessoas que sofrem de doenças crônicas) e de profissionais de saúde da linha de frente, em razão da simultaneidade e velocidade de surgimento de casos confirmados da doença. Isso contribui para que sintomas e transtornos mentais sejam desencadeados pela possibilidade de óbito, transmissibilidade e exposição ao vírus, assim como pelo impacto de todas as mudanças no funcionamento social decorrentes da pandemia (Wind, Rijkeboer, Andersson, \& Riper, 2020). Logo, o principal ponto de intervenção em crises psicológicas deve ser estabelecido pela atitude objetiva e fundamentada dos profissionais de saúde mental para contemplar as diferentes fases da epidemia (Zhang, Wu, Zhao, \& Zhang, 2020).

No Brasil, psicólogos têm se disponibilizado para prestar auxílio e acolhimento àqueles que têm sido psicologicamente afetados pela pandemia da COVID-19. Além disso, o governo tem convocado profissionais de saúde para prestar trabalho voluntário (Ministério da Saúde, 2020b). Atualmente, psicólogos e psiquiatras, para darem continuidade aos cuidados em saúde mental durante a pandemia, estão se mobilizando para realizar intervenções e atendimentos online. Medidas como essas podem ajudar a diminuir ou prevenir futuros problemas psiquiátricos e psicológicos (Cullen et al., 2020; Lima et al., 2020; Wind et al., 2020; Zhang et al., 2020).

\section{Considerações finais}

O presente artigo reuniu conhecimento científico acerca da saúde mental, da emergência do cuidado e dos aspectos associados à recentemente declarada emergência de saúde pública por conta do novo coronavírus. Foram expostos conceitos para um mais claro entendimento do cenário desencadeado pela COVID-19, bem como questões relacionadas a problemas do campo de saúde mental, fatores protetivos no desenvolvimento de transtornos mentais e, também, exemplos de possíveis intervenções em diferentes momentos da pandemia. Cabe salientar, de qualquer modo, que este trabalho não abarca, obviamente, todo o alcance da produção científica em relação ao enfrentamento de crises de saúde pública e pandemias, sendo recomendada cautela na interpretação e extrapolação das informações então apresentadas.

Espera-se que este trabalho possa contribuir para uma mais ampla avaliação do contexto em saúde mental na pandemia da COVID-19 através da perspectiva da Psicologia, especialmente a Psicologia da Saúde, e áreas afins. A partir da concepção de que a crise se apresenta por fases, o conteúdo deste artigo também pode colaborar para o desenvolvimento de programas de cuidado psicológico mais específicos para lidar com as diferentes situações que ocorrem ao longo da pandemia. Em suma, tem-se como expectativa que seja 
ressaltada a importância da compreensão do ajustamento psicológico frente às adversidades, especialmente aquelas com alto potencial de severidade e alcance, como é a COVID-19.

Na finalização deste trabalho, parece pertinente lembrar que Maunder, em 2009, escrevendo um editorial sobre o impacto psicológico em sobreviventes da SARS, comentou que o surto ocorrido em 2002, embora grave, não poderia ser considerado uma catástrofe em saúde mental. Dentre outros argumentos, o autor apontou que, como o vírus foi contido em poucas semanas e os indivíduos infectados não transmitiam a doença quando estavam assintomáticos, as medidas de restrição social foram abrandadas e a amplitude do surto foi limitada dentro de alguns países, o que minimizou o alcance de consequências mais graves sobre a saúde mental das pessoas (Maunder, 2009).

Com base na ideia de que uma epidemia pode se tornar uma catástrofe em saúde mental, e nos fatos de que, diferentemente do que ocorreu com a SARS, o novo coronavírus é propagado por pessoas ainda assintomáticas e não há expectativas claras a respeito de quando será possível controlar a disseminação, podese dizer que as proporções atualmente tomadas pela pandemia da COVID-19 (abril de 2020) se aproximam daquela definição. Entretanto, a adequação a essa noção de catástrofe só não se consolida na íntegra, neste momento, porque o evento ainda está em curso e há diferentes estágios da pandemia em diferentes países (e mesmo dentro de cada país).

O atual cenário de potencial catástrofe em saúde mental - o que requer ainda mais atenção do poder público - só será devidamente conhecido após passado o período de pandemia. Portanto, esforços imediatos devem ser empregados, em todos os níveis e pelas mais diversas áreas de conhecimento, a fim de minimizar resultados ainda mais negativos na saúde mental da população. Cabe, enfim, investir em adequada assistência à saúde e, sobretudo, na ciência em geral, para que esse período seja abreviado e que os profissionais de saúde estejam capacitados para os desafios do cuidado.

\section{Contribuição}

Todos os autores contribuíram na elaboração e revisão final do texto. A. FARO idealizou a estrutura argumentativa, participou da redação e revisão final. As demais autoras contribuíram na redação de seções específicas do texto e na revisão geral.

\section{Referências}

Agencia Estatal Boletín Oficial del Estado. (2020a). Real Decreto 463/2020, de 14 de marzo, por el que se declara el estado de alarma para la gestión de la situación de crisis sanitaria ocasionada por el COVID-19. Boletín Oficial del Estado. Madrid: Autor. Recuperado el http://www.boe.es/eli/es/rd/2020/03/14/463

Agencia Estatal Boletín Oficial del Estado. (2020b). Real Decreto 487/2020, de 10 de abril, por el que se prorroga el estado de alarma declarado por el Real Decreto 463/2020, de 14 de marzo, por el que se declara el estado de alarma para la gestión de la situación de crisis sanitaria ocasionada por el COVID-19. Boletín Oficial del Estado. Madrid: Autor. Recuperado el http://www.boe.es/eli/es/rd/2020/04/10/487

Bajardi, P., Poletto, C., Ramasco, J. J., Tizzoni, M., Colizza, V., \& Vespignani, A. (2011). Human mobility networks, travel restrictions, and the global spread of 2009 H1N1 Pandemic. Plos One, 6(1), e16591. https://doi.org/10.1371/journal. pone.0016591

Barari, S., Caria, S., Davola, A., Falco, P., Fetzer, T., Fiorin, S., ... Slepoi, F. R. (2020). Evaluating COVID-19 public health messaging in Italy: self-reported compliance and growing mental health concerns. Retrieved from http://gking.harvard. edu/covid-italy

Barbisch, D., Koenig, K. L., \& Shih, F. Y. (2015). Is there a case for quarantine? Perspectives from SARS to Ebola. Disaster Medicine and Public Health Preparedness, 9(5), 547-53. https://doi.org/10.1017/dmp.2015.38 
Bikbov, B., \& Bikbov, A. (2020). Communication on COVID-19 to community: measures to prevent a second wave of epidemic. OSF Preprints. Version 3. https://doi.org/10.31219/osf.io/ea9jm

Brooks, S. K., Webster, R. K., Smith, L. E., Woodland, L., Wessely, S., Greenberg, N., \& Rubin, G. J. (2020). The psychological impact of quarantine and how to reduce it: rapid review of the evidence. The Lancet, 395(102227), 912-920. https:// doi.org/10.1016/S0140-6736(20)30460-8

Centers for Disease Control and Prevention. (2020a). Social distancing, quarantine, and isolation: keep your distance to slow the spread. Atlanta: Author. Retrieved from http://www.cdc.gov/coronavirus/2019-ncov/prevent-getting-sick/ social-distancing.html

Centers for Disease Control and Prevention. (2020b). Severe outcomes among patients with Coronavirus Disease 2019 (COVID-19): United States, February 12-March 16, 2020. Morbidity and Mortality Weekly Report, 69(12), 343-346. https://doi.org/10.15585/mmwr.mm6912e2

Conselho Regional de Psicologia do Paraná. (2020). Nota técnica CRP-PR nº01/2020. Orienta a(o) Psicóloga(o) sobre o atendimento psicológico nas políticas públicas e instituições privadas, diante da pandemia do COVID-19. Curitiba: Autor. Recuperado de https://crppr.org.br/wp-content/uploads/2020/03/Nota-T\%C3\%A9cnica-CRP-PR-001-2020COVID19.pdf

Coronavirus Resource Center. (2020). Coronavirus COVID-19 global cases by the Center for Systems Science and Engineering (CSSE). Baltimore: Johns Hopkins University. Retrieved from http://coronavirus.jhu.edu/map.html

Cullen, W., Gulati., G., \& Kelly, B. D. (2020). Mental health in the Covid-19 pandemic. QJM: An International Journal of Medicine, 113(5), 311-312. https://doi.org/10.1093/qjmed/hcaa110

Douglas, P. K., Douglas, D. B., \& Douglas, K. M. (2009). Preparing for pandemic influenza ant its aftermath: mental health issues considered. International Journal of Emergency Mental Health, 11(3), 137-144. Retrieved from http:// www.ncbi.nlm.nih.gov/pubmed/20437844

Duan, L., \& Zhu, G. (2020). Psychological interventions for people affected by the COVID-19 epidemic. The Lancet, 7, $300-302$. https://doi.org/10.1016/\$2215-0366(20)30073-0

European Centre for Disease Prevention and Control. (2020). Considerations relating to social distancing measures in response to COVID-19: second update. Stockholm: Author. Retrieved from http://www.ecdc.europa.eu/sites/default/ files/documents/covid-19-social-distancing-measuresg-guide-second-update.pdf

Ho, C., Chee, C., \& Ho, R. (2020). Mental health strategies to combat the psychological impact of COVID-19 beyond paranoia and panic. Annals Academy Medical of Singapure, 49(3), 1-3. Retrieved from http://www.annals.edu.sg/ pdf/special/COM20043_HoCSH_2.pdf

Horton, R. (2020). Offline: COVID-19: a reckoning. The Lancet, 395(10228), 935. https://doi.org/10.1016/S01406736(20)30669-3

Inloco. (2020). Mapa brasileiro da COVID-19: índice de isolamento social. Recife: Autor. Recuperado de https://mapa brasileirodacovid.inloco.com.br/pt/?hsCtaTracking=68943485-8e65-4d6f-8ac0-af7c3ce710a2\% 7C45448575-c1a642c8-86d9-c68a42fa3fcc

Li, L.-Q., Huang, T., Wang, Y.-Q., Wang, Z.-P., Liang, Y., Huang, T.-B., ... Wang, Y. (2020). COVID-19 patients' clinical characteristics, discharge rate, and fatality rate of meta-analysis. Journal of Medical Virology, 92(6), 577-583. https:// doi.org/10.1002/jmv.25757

Li, W., Yang, Y., Liu, Z.-H., Zhao, Y.-J., Zhang, Q., Zhang, L., ... Xiang, Y.-T. (2020). Progression of mental health services during the COVID-19 outbreak in China. International Journal of Biological Sciences, 16(10), 1732-1738. https://doi. org/10.7150/ijbs.45120

Lima, C. K. T., Carvalho, P. M. M., Lima, I. A. S., Nunes, J. A. V. O., Saraiva, J. S., Souza, R. I., ... Rolim Neto, M. L. (2020). The emotional impact of coronavirus 2019-Ncov (new Coronavirus Disease). Psychiatry Research, 287, e112915. https://doi.org/10.1016/j.psychres.2020.112915

Limcaoco, R. S. G., Mateos, E. M., Fernandez, J. M., \& Roncero, C. (2020). Anxiety, worry and perceived stress in the world due to the COVID-19 pandemic: preliminary results. MedRxivPreprint. https://doi.org/10.1101/2020.04.03.20043992

Lung, F. W., Lu, Y. C., Chang, Y. Y., \& Shu, B. C. (2009). Mental symptoms in different health professionals during the SARS attack: a follow-up study. Psychiatric Quarterly, 80(2), 107-116. https://doi.org/10.1007/s11126-009-9095-5

Mahase, E. (2020). Coronavirus: COVID-19 has killed more people than SARS and MERS combined, despite lower case fatality. The BMJ, 368, m641. https://doi.org/10.1136/bmj.m641

Maunder, R. G. (2009). Was SARS a mental health catastrophe? General Hospital Psychiatry, 31(4), 316-317. http:// dx.doi.org/10.1016/j.genhosppsych.2009.04.004 
Ministério da Saúde (Brasil). (2020a). Plano de contingência nacional para infecção humana pelo novo Coronavírus 2019-nCoV: centro de operações de emergências em saúde pública (COE-nCoV). Braślia: Autor. Recuperado de http://portalarquivos2. saude.gov.br/images/pdf/2020/fevereiro/07/plano-contingencia-coronavirus-preliminar.pdf

Ministério da Saúde (Brasil). (2020b). Saúde mental e atenção psicossocial na COVID-19: um guia para gestores. Fiocruz: Autor. Recuperado de http://www.fiocruzbrasilia.fiocruz.br/wp-content/uploads/2020/04/Sa\%c3\%bade-Mental-eAten\%c3\%a7\%c3\%a3o-Psicossocial-na-Pandemia-Covid-19-recomenda\%c3\%a7\%c3\%b5es-para-gestores.pdf

Ministério da Saúde (Brasil). (2020c). Portaria № 454, de 20 de março de 2020. Declara, em todo o território nacional, o estado de transmissão comunitária do coronavírus (covid-19). Diário Oficial da União. Brasília: Autor. Recuperado de http://www.in.gov.br/en/web/dou/-/portaria-n-454-de-20-de-marco-de-2020-249091587

Ministero della Salute (Italia). (2020). Tougher penalties for those who do not respect the restrictive measures [in Italian]. Retrieved from http://www.salute.gov.it/portale/nuovocoronavirus/dettaglioNotizieNuovoCoronavirus. jsp?lingua=italiano\&menu=notizie $\& p=$ dalministero $\& i d=4324$

National Health Commission of China (2020a). Principles of the emergency psychological crisis interventions for the new coronavirus pneumonia [in Chinese]. Retrieved from http://www.nhc.gov.cn/jkj/ s3577/202001/6adc08b966594253b2b791be5c3b9467

National Health Commission of China (2020b). The National Health and Health Commission held a video and telephone conference deploying the next stage of prevention and control according to law, scientific prevention and control, precise prevention and control, and resolutely prevent the input of overseas epidemic situations [in Chinese]. Retrieved from http://www.nhc.gov.cn/bgt/s7693/202003/34e057348f244b0f9391d569334b844e.shtml

Onder, G., Rezza, G., \& Brusaferro, S. (2020). Case-fatality rate and characteristics of patients dying in relation to COVID-19 in Italy. JAMA, 323(18), 1775-1776. https:///doi.org/10.1001/jama.2020.4683

Ozili, P., \& Arun, T. (2020). Spillover of COVID-19: impact on the global economy. SSRN Preprints. https://doi.org/10.2139/ ssrn.3562570

Pancani, L., Marinucci, M., Aureli, N., \& Riva, P. (2020). Forced social isolation and mental health: a study on 1006 Italians under COVID-19 quarantine. PsyArXiv Preprints. https://doi.org/10.31234/osf.io/uacfj

Park, S., \& Park, Y. C. (2020). Mental health care measures in response to the 2019 novel coronavirus outbreak in Korea. Psychiatry Investigation, 17(2), 85-86. https://doi.org/10.30773/pi.2020.0058

Peeri, N. C., Shrestha, N., Rhaman, M. S., Zaki, R., Tan, Z., Bibi, S., ... Haque, U. (2020). The SARS, MERS and novel coronavirus (COVID-19) epidemics, the newest and biggest mental health threats: what lessons have we learned? International Journal of Epidemiology, O(0), 1-10. https://doi.org/10/1093/ije/dyaa033

Presidência da República (Brasil). (2020). Lei n 13.979, de 6 de fevereiro de 2020. Dispõe sobre as medidas para enfrentamento da emergência de saúde pública de importância internacional decorrente do coronavírus responsável pelo surto de 2019. Diário Oficial da União. Brasília: Autor. Recuperado de http://www.in.gov.br/en/web/dou/-/lei-n13.979-de-6-de-fevereiro-de-2020-242078735

Qiu, J., Shen, B., Zhao, M., Wang, Z., Xie, B., \& Xu, Y. (2020). A nationwide survey of psychological distress among Chinese people in the COVID-19 epidemic: implications and policy recommendations. General Psychiatric, 33(2), e100213. https://doi.org/10.1136/gpsych-2020-100213

Raude, J., Debin, M., Souty, C., Guerrisi, C., Turbelin, C., Falchi, A., ... Duggan, J. (2020). Are people excessively pessimistic about the risk of coronavirus infection? PsyArXiv Preprints. https://doi.org/10.31234/osf.io/364qj

Remuzzi, A., \& Remuzzi, G. (2020). COVID-19 and Italy: what next? The Lancet, 395, 1225-1228. https://doi.org/10.1016/ S0140-6736(20)30627-9

Sohrabi, C., Alsafi, Z., O’Neil, N., Khan, M., Kerwan, A., Al-Jabir, A., ... Agha, R. (2020). World Health Organization declares global emergency: a review of the 2019 novel coronavirus (COVID-19). International Journal of Surgery, 76, 71-76. https://doi.org/10.1016/j.ijsu.2020.02.034

Stevenson, E., Barrios, L., Cordell, R., Deloizier, D., Gorman, S., Koenig, L. J., ... Singleton, C. (2009). Pandemic influenza planning: addressing the needs of children. American Journal of Public Health, 99(2), 255-260. Retrieved from http:// ajph.aphapublications.org/doi/pdf/10.2105/AJPH.2009.159970

van Bavel, J. J., Baicker, K., Boggio, P., Capraro, V., Cichocka, A., Cikara, M., ... Willer, R. (2020). Using social and behavioral science to support COVID-19 pandemic response. PsyArXiv Preprints. https://doi.org/10.31234/osf.io/y38m9

Wang, C., Pan, R., Wan, X., Tan, Y., Xu, L., Ho, C. S., \& Ho, R. C. (2020). Immediate psychological responses and associated factors during the initial stage of Coronavirus Disease (COVID-19) epidemic among the general population in China. International Journal of Environmental Research and Public Health, 17(5), 1729. http://dx.doi.org/10.3390/ ijerph17051729 
Wang, G., Zhang, Y., Zhao, J., Zhang, J., \& Jiang, F. (2020). Mitigate the effects of home confinement on children during the COVID-19 outbreak. The Lancet, 395, 945-947. http://dx.doi.org/10.1016/S0140-6736(20)30547-X

Weissman, G. E., Crane-Droesch, A., Chivers, C., ThaiBinh, L., Hanish, A., Levy, M. Z., ... Halpern, S. D. (2020). Locally informed simulation to predict hospital capacity needs during the COVID-19 pandemic [Ahead of print]. Annals of Internal Medicine. https://doi.org/10.7326/M20-1260

Wind, T. R., Rijkeboer, M., Andersson, G., \& Riper, H. (2020). The COVID-19 pandemic: the 'black swan' for mental health care and a turning point for e-health. Internet Interventions, 20, e10317. http://dx.doi.org/10.1016/j.invent.2020.100317

World Health Organization. (2007). Risk reduction and emergency preparedness: World Health Organization six-year strategy for the health sector and community capacity development. Geneva: Author. Retrieved from http://apps.who.int/iris/bitstream/ handle/10665/43736/9789241595 896_eng.pdf?sequence=1\&isAllwed=y

World Health Organization. (2020a). Coronavirus disease 2019 (COVID-19): situation report - 78. Geneva: Author. Retrieved from http://www.who.int/docs/default-source/coronaviruse/situation-reports/20200407-sitrep-78-covid-19.pdf?sfvrsn=bc43e1b_2

World Health Organization. (2020b). Report of the WHO-China joint mission on coronavirus disease 2019 (COVID-19). Geneva: Author. Retrieved from http://www.who.int/docs/default-source/coronaviruse/who-china-joint-mission-oncovid-19-final-report.pdf

Xiang, Y. T., Yang, Y., Li, W., Zhang, L., Zhang, Q., Cheung, T., ... Ng, C. H. (2020).Timely mental health care for the 2019 novel coronavirus outbreak is urgently. The Lancet, 7(3), 228-229. https://doi.org/10/1016/S22150366(20)30046-8

Yang, Y., Li, W., Zhang, Q., Zhang, L., Cheung, T., \& Xiang, Y. T. (2020). Mental health services for older adults in China during the COVID-19 outbreak. The Lancet, 7, e19. https://doi.org/01016/S2215-0366(20)30079-1

Zhai, Y., \& Du, X. (2020). Mental health care for international Chinese students affected by the COVID-19 outbreak. The Lancet, 7 , e22. https://doi.org/10.1016/S2215-0366(20)30089-4

Zhang, J., Wu, W., Zhao, X., \& Zhang, W. (2020). Recommended psychological crisis intervention response to the 2019 novel coronavirus pneumonia outbreak in China: a model of West China Hospital. Precision Clinical Medicine, 3(1), 3-8. https://doi.org/10.1093/pcmedi/pbaa006 